if the orbit is assumed to be a parabola, and by solving

$$
\left(z^{2}-2 c z+1\right)^{3}(z-m)^{3}-m^{2}=0
$$

if no assumption is made regarding the orbit. One of the chief aims of Professor Leuschner's short methods for computing an orbit is to shorten the time taken for the calculation. The semi-graphical solution of the above equations used in the short methods contributes largely to this end, as well as to the lessening of tedious computational work; but this method still requires the use of tables. It is to eliminate entirely all computations in the solution of equation (1) above that Professor Leuschner and Mr. Bernstein devised methods of constructing geometrically the curve given by the equation

$$
y=\frac{h}{\left[(z-c)^{2}+s^{2}\right]^{\frac{1}{2}}},
$$

whose intersections with the parabola $y=\left(z-p^{\prime}\right)^{2}$ give the required roots. By finding a geometric construction of the curve

$$
z-m=\frac{m}{\left(z^{2}-2 c+1\right)^{\frac{3}{2}}},
$$

whose intersections with the line $m y=x+c-m$ give the roots of equation (2), Mr. Bernstein derived a purely graphical solution of the problem for the general orbit.

$$
\begin{gathered}
\text { T. M. Putnam, } \\
\text { Secretary of the Section. }
\end{gathered}
$$

\title{
THE CARLSRUHE MEETING OF THE GERMAN MATHEMATICAL SOCIETY.
}

THE annual meeting of the Deutsche Mathematiker-Vereinigung was held in affiliation with the eighty-third convention of the society of naturalists and physicians at Karlsruhe during the week of September 24-28, under the presidency of Professor F. Schur, of the University of Strassburg. Under the stimulus of the pleasure and profit derived from preceding meetings a new record in attendance was established, over one hundred persons being present. The people of Karlsruhe generously 
provided for the social part of the meeting by arranging an excellent program for every evening, one being devoted to an excursion to Heidelberg to witness the illumination of the castle, and to hear a well-rendered concert. The general session of Monday morning was opened with an address of welcome by the Grand Duke of Baden. Sectional meetings were held during the next four days, and the business meeting on Thursday morning. The membership of the society is 765 and the reserve fund consists of over M. 23,000. The bibliographic commission has been discontinued, and new members have been added to the committee to provide for the further publication of the papers of E. Schröder. Two finished volumes of the works of Euler were presented, and a detailed report of the German subcommittee of the international commission of mathematical instruction was read. Professors Rohn and Sommerfeld succeed Professors Engel and Schur as members of the council. The official representatives to the fifth congress of mathematicians are the new chairman, Professor W. von Dyck, and the secretary, Professor M. Krazer. The next meeting of the Vereinigung will be held at Münster in September, 1912, under the presidency of Professor von Dyck.*

The following papers were read at this meeting.

(1) Professor C. Carathéodory, Breslau: "On the depiction of the boundary in the conformal representation of a general region."

(2) Professor O. Blumenthal, Aachen: "Meromorphic functions of several variables."

(3) Professor G. FABER, Stuttgart: "On interpolation."

(4) Professor L. G. Du PAsquier, Neuenburg: "On complex tettarions."

(5) Dr. L. Lichtenstein, Berlin: “On Poisson's integral and the behavior of analytic functions on the boundary of the circle of convergence."

(6) Professor L. Schlesinger, Giessen: "On early work of Gauss on the arithmetic-geometric mean."

(7) Professor K. Heun, Karlsruhe: "Outlines for the extension of classical mechanics" (report).

(8) Professor P. Voronetz, Kieff: "On the equations of motion of a rigid body."

\footnotetext{
* For this part of the report the editors are indebted to Dr. H. MoHrMaNN, of Karlsruhe; for that of the social activities, to Dr. Clara A. SmITH. The abstracts of the paper were kindly furnished by the authors in reply to a request sent by the editors.
} 
(9) Professor R. v. Mises, Strassburg: "On the problems of oscillation and the Sommerfeld theorem of turbulence."

(10) Professor G. Hamel, Brünn: "On the stiffness of ropes."

(11) Professor V. VARICAK, Agram: "On the non-euclidean interpretation of the theory of relativity."

(12) Professor E. Pappenitz, Freiberg: "Exhibition of the completed kinodiaphragmatic projection apparatus for geometric illustrations.'

(13) Professor H. Wiener, Darmstadt: "Determination of a root of an equation by means of a convergent (or divergent) series."

(14) Professor E. Salkowski, Charlottenburg: "Theory of curves in elliptic space."

(15) Professor C. Juel, Copenhagen: "Concerning the straight lines which lie on an analytic non-algebraic surface of order three."

(16) Professors F. Klein, Göttingen, E. Brouwer, Amsterdam, and P. Koebe, Leipzig: "Report on automorphic functions and the problem of uniformization."

(17) Professor E. HrLB, Würzburg: "Connection between fundamental regions and linear differential equations."

(18) Dr. L. Bieberbach, Königsberg: "Remarks on automorphic functions."

(19) Professor J. Wellstein, Strassburg: "On motion in space and the principle of relativity."

(20) Professor L. Heffter, Freiburg: "On the introduction of the four-dimensional world of Minkowski."

(21) Professor F. Bernstern, Göttingen: "On Lagrange's problem of mean motion in the theory of secular perturbations."

(22) Professor H. Wiener, Darmstadt: "Exhibition and explanation of some new models."

Abstracts of several of the papers follow; the numbers correspond to those in the list above.

2. As an extension of memoirs by E. E. Levi, Professor Blumenthal discussed the natural boundaries of functions of two complex variables. He showed by means of the principle of condensation that the natural boundaries approach certain three-dimensional manifolds, many of the properties of which can be ascertained. The memoir is to appear in the Jahresbericht.

3. Professor Faber spoke of his investigations concerning 
the accuracy of the best formulas of interpolation, and pointed out that they were less applicable to the representation of a function than to obtaining numerical approximations in mechanical quadrature. At the conclusion of the discussion the methods of Tchebichef and of Cauchy were compared.

4. The tettarions discussed by Professor Du Pasquier are defined as matrices of numbers having the same number of rows as of columns. They are added or subtracted by adding or subtracting corresponding components. Multiplication follows the same laws as the composition of linear substitutions. As subgroups, the complex numbers of Gauss and the quaternions of Hamilton are included. If $\nu=4$ and all the components are real rational numbers, a tettarion field is defined. Division into integral and fractional forms can be made in an unlimited number of ways. Thus an infinite number of realms of rationality exist. A rational basis of classification is into integral, conditionally fractional, unconditionally fractional, and finally forms having both characters.

5. In the paper of Dr. Lichtenstein a number of properties of the partial derivatives of the Poisson integral, taken on the circumference, are derived. It is then shown that if $u(x, y)$ is a Poisson integral with integrable boundary function and $v(x, y)$ its complementary potential function, then the latter is integrable. The most important result concerns the partial derivatives of the second order of the logarithmic potential

$$
u(x, y)=\frac{1}{4 \pi} \iint_{T} \log \left\{(x-\xi)^{2}+(y-\eta)^{2}\right\} P(\xi, \eta) d \xi d \eta
$$

in which $P(\xi, \eta)$ is an integrable function and $T$ any finite region. It is shown that except for a set of points of content zero, the derivatives $\partial^{2} u / \partial x^{2}, \partial^{2} u / \partial y^{2}$ exist, are integrable, and satisfy the equation

$$
\frac{\partial^{2} u}{\partial x^{2}}+\frac{\partial^{2} u}{\partial y^{2}}=P(x, y)
$$

8. The differential equations of motion of a rigid body are usually referred to a fixed system $O_{1}-x_{1} y_{1} z_{1}$ of rectangular coordinates, or to a system $O-x y z$ moving rigidly with the body. In the few cases in which a system $M-\xi \eta \zeta$ is employed which is movable as to both, $M$ is either supposed fixed, or is taken as the center of gravity of the moving body. 
In Professor Voronetz's paper, the first part is concerned with the equations of motion in $M-\xi \eta \zeta$ coordinates, the position of $M$ being unrestricted; the second part develops the case of rolling motion further, and contains a number of new results.

11. Professor Varicak showed in his paper that the Lobachevsky geometry furnishes adequate means for the treatment of the theory of relativity; all of the investigations operate in a Lobachevsky space. By means of simple considerations, the addition theory for velocities is developed, as well as the laws of aberration, of reflection in moving mirrors, and certain other laws.

12. Professor Papperitz presented an improved complete form of his apparatus for geometric illustrations. For the principle of the procedure and its applicability for producing figures in space for the purposes of instruction, reference may be made to two short reports ("Ueber das Zeichnen in Raume," Jahresbericht, volume 19 (1911), page 307, and "Die kinodiaphragmatische Projection . . . ,"Hoffmann's Zeitschrift, volume 11 (1911), page 465). This method of illustration promises to be particularly expedient; it represents not only figures in a plane, but also stereometric ones by means of virtual images of space curves and surfaces. The apparatus has been patented, and is provided with a number of models for representation. A detailed prospectus is furnished with the machine.

14. The principle of correspondence introduced by Study (American Journal, volume 29, pages $116 \mathrm{ff}$.) by means of which the speers of elliptic space are associated with the point-spheres of two spheres of unit radius in euclidean space is applied by Professor Salkowski to the theory of space curves. If $x_{l_{1}}$ $x_{l_{2}}, x_{l_{3}}, x_{r_{1}}, x_{r_{2}}, x_{r_{3}}$ are the speer coordinates of the directed tangent of a space curve, $y_{l_{i}}, y_{r_{i}} ; z_{l_{i}}, z_{r_{i}}(i=1,2,3)$ those of its principal normal and binormal respectively, $\kappa, \tau$ the curvature and torsion, then the equations exist

$$
\begin{gathered}
\frac{d x_{l_{i}}}{d s}=\kappa y_{l i}, \quad \frac{d x_{r_{i}}}{d s}=\kappa y_{r_{i}}, \\
\frac{d y_{l_{i}}}{d s}=-\kappa x_{l_{i}}-(\tau-1) x_{l_{i}}, \quad \frac{d y_{r_{i}}}{d s}=-\kappa x_{r_{i}}-(\tau+1) z_{r_{i}}, \\
\frac{d z_{l_{i}}}{d s}=(\tau-1) y_{r_{i}}, \quad \frac{d z_{r_{i}}}{d s}=(\tau+1) y_{r_{i}}
\end{gathered}
$$


These equations determine a curve of elliptic space, and, except as to a simple transformation, associate two curves $c_{l}, c_{r}$ of euclidean space. A number of consequences of this theorem were pointed out.

16. The report on automorphic functions was presented by Professor Koebe. The most emphasis was put on the problem of uniformization, and a statement of recent progress made was given. After discussing his own publications (Jahresbericht, for 1906, 1907, 1910; Göttinger Nachrichten, 1907-1911; Mathematische Annalen, volumes 67, 69; Crelle's Journal, volumes 138, 139), the speaker pointed out the significance of recent memoirs of Poincaré, Hilbert, and others.

17. If four singular (real) points and their corresponding exponents of a linear differential equation of the second order are known, there are $\infty^{1}$ real values of the parameter, so that the circular quadrilateral has an orthogonal circle. In case of complex parameters, an infinite number of new theorems can be stated, one associated with each characteristic oscillation number. To generalize the above conditions, Professor Hilb uses the relations established by Klein for the triangle and by Ihlenberg for the quadrilateral. It is shown that the method developed by the latter becomes too complicated to be further extended. The author showed how to pass from polygons of $n$ sides to those of $n+1$ sides by a new method. The corresponding extension in the complex field is much more complicated, but can be made. By means of the Fricke normal polygons, it is possible to overcome the difficulty imposed by the Stieltjes boundary, making the transformation of $y^{\prime}$ (or higher derivative) for $y$, then taking the auxiliary points into account.

18. Dr. Bieberbach reported on the contents of the third installment of Fricke-Klein, Vorlesungen über automorphe Funktionen, and made some remarks concerning the proof by means of continuity of the theorem of the closed section.

VIRGIL SNYDER. 